\title{
Penile Metastasis of Colorectal Cancer Mimicking Priapism with Acute Urinary Retention: A Rare Case
}

\author{
Grace Lum Shi Xian ${ }^{1}$, Lit Kiat Tan², George Eng Geap Lee ${ }^{3}$ \\ ${ }^{1}$ Universiti Kebangsaan Malaysia, Kuala Lumpur, Malaysia \\ ${ }^{2}$ University of Dundee, Scotland, United Kingdom \\ ${ }^{3}$ Monash University, Sunway Campus, Bandar Sunway, Malaysia \\ Email: ^grace_lum95@hotmail.com
}

How to cite this paper: Xian, G.L.S., Tan, L.K. and Lee, G.E.G. (2021) Penile Metastasis of Colorectal Cancer Mimicking Priapism with Acute Urinary Retention: A Rare Case. Open Journal of Urology, 11, 1-5. https://doi.org/10.4236/oju.2021.111001

Received: November 24, 2020

Accepted: January 11, 2021

Published: January 14, 2021

Copyright $\odot 2021$ by author(s) and Scientific Research Publishing Inc. This work is licensed under the Creative Commons Attribution International License (CC BY 4.0).

http://creativecommons.org/licenses/by/4.0/

\begin{abstract}
Background: Penile metastasis of colorectal carcinoma is a rare phenomenon in clinical setting. They normally manifest as penile lesion and acute urinary retention. However, presentation of priapism is exceedingly rare. Aims: Discussion of this rare presentation as well as the diagnostic processes and subsequent management. Case Presentation: A 54-year-old male with a history of colorectal cancer presents with acute urinary retention. Examination of the patient demonstrates a semi-erect penis, with multiple palpable nodules on the shaft and penile meatus. Histological and imaging findings indicate penile metastasis of colorectal cancer. Conclusion: Biopsy via cystoscopy is used to obtain definitive diagnosis of penile metastasis. Urinary drainage followed by further cancer intervention or palliative care is crucial for effective management.
\end{abstract}

\section{Keywords}

Metastatic Colorectal Cancer, Urinary Retention, Priapism, Secondary Penile Malignancy

\section{Introduction}

Colorectal carcinoma is a common malignancy affecting large demographic of populations across the world. Secondary colorectal cancers are also well documented in literature, with common sites of metastasis to the lungs, liver, bones and brain. Although prostate and penile tissues are in close proximity to sigmoid colon and have an extensive circulatory connection, metastases of colorectal cancer to them are rare. The first reported case was by Eberth in 1870 when he 
reported penile metastasis from the rectum. In total, there have been about 400 cases of penile malignancy due to metastasis reported with only less than 60 cases related to colorectal cancer to have ever been reported in literatures [1] [2] [3]. Penile metastasis of colorectal cancer has clinical manifestation, such as penile nodules, priapism, urinary retention, and skin nodules. However, presentation of skin nodules and priapism are exceptionally rare [4] [5]. We present a rare case of such metastasis mimicking priapism, resulting in acute urinary retention. The diagnostic process and subsequent management are also discussed.

\section{Case Presentation}

A 54-year-old male with a history of colorectal cancer, who had undergone hemicolectomy 2 years ago, presents with acute urinary retention. The histological and post-operative imaging taken during his initial diagnosis 2 years ago revealed a T2 adenocarcinoma with no evidence of lymph node involvement (T2NOM0). Present examination of the patient demonstrates a semi-erect penis, with multiple palpable nodules on the shaft and penile meatus. Urethral catheterization was not possible due to the persistent erection and occluding metastatic nodules. Intra-cavernosal aspiration of penis arterial blood sample was analysed, demonstrating no evidence of high or low flow priapism. Pelvic CT scans also confirmed multiple lesions in corpus cavernosum, likely derived from the local recurrence of the colorectal cancer Figure 1. Doppler Ultrasound study of the patient shows no vascular abnormality of the caversonal arteries of corpus cavernosum and corpus spongiosum which excludes priapism. The ultrasound imaging also revealed multiple ill-defined lesions invading corpus cavernosum as well as on the subcutaneous tissues Figure 2. The patient subsequently underwent emergency cystoscopy to facilitate urethral drainage, relieving the patient of the retention of urine as well as obtaining subcutaneous and urethral penile samples for biopsy to aid the diagnosis. The histological analysis with haematoxylin and eosin stained of the urethral biopsy revealed tissue lined by urothelial epithelium with underlying malignant tumour and lympho-vascular permeation. Specimen from exterior penile biopsy shows ulcerated mucosa (stratified squamous epithelium)

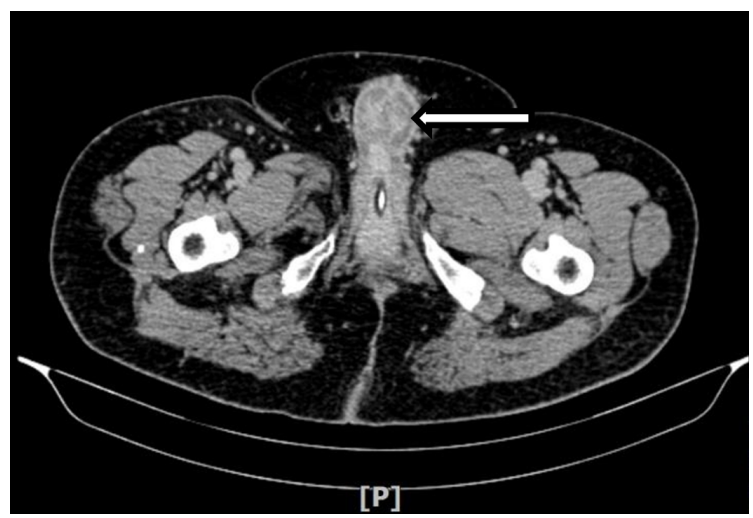

Figure 1. Pelvic CT scan showing marked thickening and heterogenous enhancement within corpus cavernosa. 
with moderate neutrophils infiltration. Further immunohistochemistry differentiation also confirmed specimens to be CDX 2 and CK 20 positive, while CK 7 and PSA were negative Figure 3. The histological analysis definitively indicates that both lesions originated from the metastasis of colorectal carcinoma. Following confirmative diagnosis, the patient underwent abdomino-perineal resection followed by 6 months of adjuvant chemotherapy, Xeloda. Patient's routine CT scan and colonoscopy was done 6 months after the end of chemotherapy which shows no recurrences.

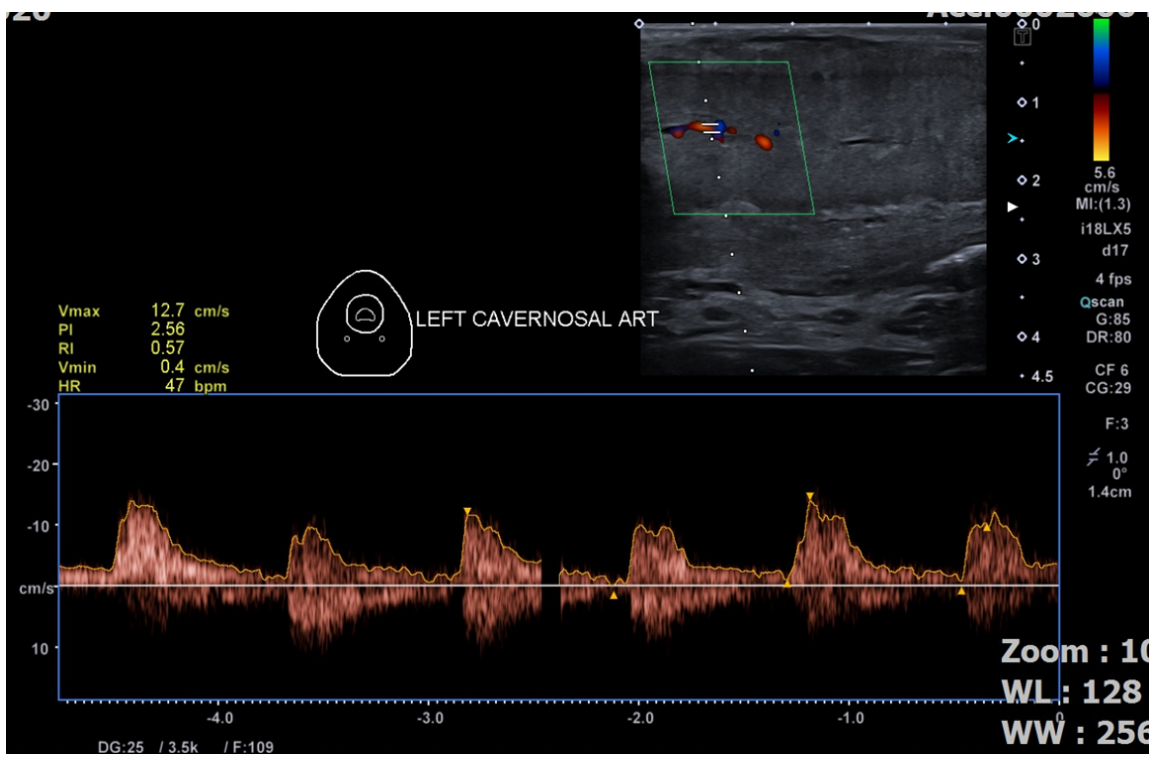

Figure 2. Doppler Ultrasound multiple ill-defined masses in corpus cavernosum bilaterally and subcutaneous tissues.

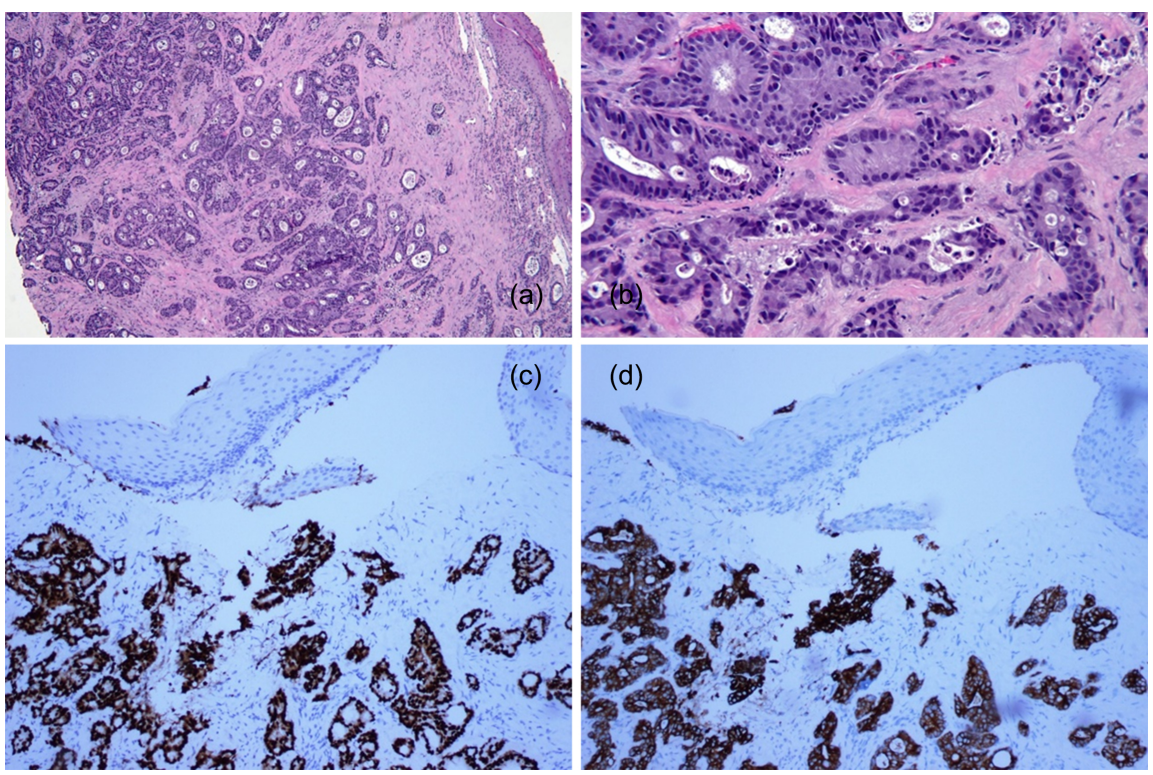

Figure 3. (a) H\&E staining on urethral biopsy specimen; Lympho-vascular permeation is noted (original magnification $\times 100$ ); (b) H\&E staining on penile biopsy specimen; No Pagetoid spread seen (original magnification $\times 400$ ); (c) CDX2 positive; (d) CK20 positive. 


\section{Discussion}

Penile metastasis was first described by Eberth in 1870 [3]. Since the initial publications, only 400 such cases were recorded in literature. The penis is a rare site for metastasis. The stipulated mechanisms of metastasis can either be by venous route, lymphatic system, arterial spread, direct extension or by iatrogenic implantation as described by Paquin and Roland in 1956 [2] [5]. As a result of rich communication between the dorsal penile venous system and the pelvic organ, venous spread is the most likely mechanism of metastasis [6].

The clinical manifestation associated with penile metastasis are as acute urinary retention, priapism, penile nodules, skin nodules, generalize swelling, and oedema [3]. The penile nodules presented in secondary penile malignancy are usually deep within the corpus cavernosa rather than superficially like primary penile cancers. Imaging modalities such as Ultrasound, CT and MRI scans are ideal non-invasive methods to evaluate characteristics of lesion.

The presentation mimicking priapism in this case is believed to be due to neoplastic invasion of the corpus cavernosum which has cause impairment of venous return of the penis, causing accumulation of blood at the penis. Intra-cavernosal aspiration of penis arterial blood sample was analysed, demonstrating no evidence of high or low flow priapism. The patient had also presented with urinary retention, attempts of catherization to relieve the retention was not possible due to neoplastic lesion causing a blockage, restricting the access for the catheter.

As part of emergency management, cystoscopy is highly recommended as the procedure relieves the patient of retention of urine as well as providing an opportunity to obtain biopsy samples as the only definitive method of diagnosing penile metastasis requires fine-needle aspiration biopsy of the lesion for further histopathology and immunohistochemistry confirmation [3]. It is important to note that the process of diagnosing a patient with penile metastasis are by the process of elimination as it is crucial to first exclude other differential diagnosis such as primary penile cancer, chancre, chancroid, non-tumorous priapism, Peyronie's disease, tuberculosis and other inflammatory and suppurative diseases [1] [2].

The prognosis of the patient is generally described as poor as overall survival for patients with secondary penile malignancy is approximately nine month with one study describing $100 \%$ mortality rate [4]. Treatment plan may vary depending on the general health of the patient, as well as the site of primary, extent of metastatic spread and the severity of symptoms [2]. Options includes local excision, penectomy, chemotherapy and radiotherapy. However due to the poor prognosis of secondary penile malignancy, Palliative treatment and improvement of quality of life are main treatments for these patients. Invasive treatments such as partial or complete penectomy are not suggested because the survival rate enhancement is unremarkable, except in patients with small lesions which may yield a positive outcome [5]. The primary aim in the management of patient with metastasis penile malignancy is early detection, precise diagnosis and 
non-invasive treatment for improvement of quality of life [1].

\section{Conclusion}

Penile metastasis of colorectal cancer is a rare phenomenon as there have only been around 400 cases reported since 1870 . However, penile metastasis that mimics priapism resulting in acute urinary retention has not been previously reported. Non-invasive investigation such as imaging is ideal to evaluate the penile lesion and exclusion of the possibility of priapism. Fine-needle aspiration biopsy is required for definitive histopathology and immunohistochemistry confirmation. Following definitive diagnosis, urinary drainage is necessary to relieve urinary retention followed by further cancer intervention or palliative management to improve the quality of life of the patient. The prognosis for metastatic penile malignancy is poor.

\section{Statement of Informed Consent}

A verbal informed consent was obtained from the patient for the information and images used in this publication.

\section{Conflicts of Interest}

The authors declare no conflicts of interest regarding the publication of this paper.

\section{References}

[1] Park, J.C., Lee, W.H., Kang, M.K. and Park, S.Y. (2009) Priapism Secondary to Penile Metastasis of Rectal Cancer. World Journal of Gastroenterology, 15, 4209-4211. https://doi.org/10.3748/wjg.15.4209

[2] Cherian, J., et al. (2006) Secondary Penile Tumours Revisited. International Seminars in Surgical Oncology, 3, No. 1. https://doi.org/10.1186/1477-7800-3-33

[3] Sibarani, J., et al. (2020) Malignant Priapismus Induced by Adenocarcinoma of the Prostate. Urology Case Reports, 29, Article ID: 101102. https://doi.org/10.1016/j.eucr.2019.101102

[4] Lin, Y.H., Kim, J.J., Stein, N.B. and Khera, M. (2011) Malignant Priapism Secondary to Metastatic Prostate Cancer: A Case Report and Review of Literature. Reviews in Urology, 13, 90-94

[5] Mearini, L., Colella, R., Zucchi, A., Nunzi, E., Porrozzi, C. and Porena, M. (2012) A Review of Penile Metastasis. Oncology Reviews, 6, No. 1. https://doi.org/10.4081/oncol.2012.e10

[6] Adeyoju, A.B., et al. (2002) Priapism in Sickle-Cell Disease; Incidence, Risk Factors and Complications-An International Multicentre Study. BJU International, 90, 898-902. https://doi.org/10.1046/j.1464-410X.2002.03022.x 